\title{
Rubidium-82 PET imaging is feasible in a rat myocardial infarction model
}

\author{
Adam Ali Ghotbi, MD, ${ }^{a}$ Andreas Clemmensen, MSc, ${ }^{a}$ Kasper Kyhl, $M D,{ }^{b}$ \\ Bjarke Follin, MSc, ${ }^{\text {a,c }}$ Philip Hasbak, MD, DMSc, ${ }^{\text {a }}$ Thomas Engstrøm, MD, DMSc, ${ }^{b}$ \\ Rasmus Sejersten Ripa, MD, DMSc, ${ }^{a}$ and Andreas Kjaer, MD, PhD, DMSc ${ }^{a}$ \\ a Department of Clinical Physiology, Nuclear Medicine \& PET and Cluster for Molecular Imaging, \\ Rigshospitalet and University of Copenhagen, Copenhagen, Denmark \\ b Department of Cardiology, The Heart Center, Rigshospitalet and University of Copenhagen, \\ Copenhagen, Denmark \\ c Cardiology Stem Cell Center, The Heart Center, Rigshospitalet and University of Copenhagen, \\ Copenhagen, Denmark
}

Received Mar 27, 2017; accepted Jun 29, 2017

doi: 10.1007/s12350-017-0994-9

Background. Small-animal myocardial infarct models are frequently used in the assessment of new cardioprotective strategies. A validated quantification of perfusion using a noncyclotron-dependent PET tracer would be of importance in monitoring response to therapy. We tested whether myocardial PET perfusion imaging is feasible with Rubidium-82 $\left({ }^{82} \mathrm{Rb}\right)$ in a small-animal scanner using a rat myocardial infarct model.

Methods. 18 Sprague-Dawley rats underwent permanent coronary artery ligation (infarct group), and 11 rats underwent ischemia-reperfusion (reperfusion group) procedure. ${ }^{82} \mathrm{Rb}$-PET and magnetic resonance imaging (MRI) were conducted before and after the intervention. Perfusion was compared to both left ventricle ejection fraction (LVEF) and infarct size assessed by MRI.

Results. Follow-up global ${ }^{82} \mathrm{Rb}$-uptake correlated significantly with infarct size (infarct group: $r=-0.81, P<0.001$ and reperfusion group: $r=-0.61, P=0.04)$. Only ${ }^{82} \mathrm{Rb}$-uptake in the infarct group correlated with LVEF. At follow-up, a higher segmental ${ }^{82} \mathrm{Rb}$-uptake in the infarct group was associated with better wall motion $(\beta=0.034$, CI $[0.028 ; 0.039], P<0.001$, $\left.R^{2}=0.30\right)$, and inversely associated with scar transmurality $(\beta=-2.4[-2.6 ;-2.2]$, $P<0.001, R^{2}=0.59$ ). The associations were similar for the reperfusion group.

Conclusion. ${ }^{82} \mathrm{Rb}$-PET is feasible in small animal scanners despite the long positron range and enables fast and time-efficient myocardial perfusion imaging in rat models. ( $\mathrm{J}$ Nucl Cardiol 2019;26:798-809.)

Key Words: Small-animal heart $\cdot$ rat myocardium $•$ perfusion imaging $\cdot$ infarction • rubidium-82 PET $\cdot$ magnetic resonance

Electronic supplementary material The online version of this article (doi:10.1007/s12350-017-0994-9) contains supplementary material, which is available to authorized users.

The authors of this article have provided a PowerPoint file, available for download at SpringerLink, which summarises the contents of the paper and is free for re-use at meetings and presentations. Search for the article DOI on SpringerLink.com.

Funding No relationship or funding from the industry.
Reprint requests: Adam Ali Ghotbi, MD, Department of Clinical Physiology, Nuclear Medicine \& PET and Cluster for Molecular Imaging, Rigshospitalet and University of Copenhagen, Blegdamsvej 9, 2100 Copenhagen, Denmark; adamghotbi@gmail.com $1071-3581 / \$ 34.00$

Copyright (C) 2017 The Author(s). This article is an open access publication 


\begin{tabular}{|ll|}
\hline Abbreviations \\
AHA-17 & American Heart Association 17 segment \\
& model \\
CT & Computed tomography \\
EDV & End-diastolic volume \\
ESV & End-systolic volumes \\
LAD & Left anterior descending artery \\
LGE & Late gadolinium enhancement \\
LV & Left ventricle \\
LVEF & Left ventricle ejection fraction \\
MI & Myocardial infarction \\
MRI & Magnetic resonance \\
OSEM & Ordered-subsets expectation \\
& maximization \\
PET & Positron emission tomography \\
ROI & Region of interest \\
TR & Repetition time \\
TE & Echo time \\
SPECT & Single-photon emission computed \\
& tomography \\
${ }^{82}$ Rb & Rubidium-82 \\
\hline
\end{tabular}

See related editorial, pp. 810-813

\section{INTRODUCTION}

Small-animal heart models, e.g., rat or mouse, are widely used to explore pathophysiology and test new cardioprotective modalities in the infarct and ischemiareperfusion settings. ${ }^{1,2}$ To this end, it is necessary to establish the infarct area. Histological techniques are available, but their application is limited since multiple, and longitudinal assessments of the myocardial injury are not possible. ${ }^{3}$ Non-invasive techniques are preferred because they allow serial assessment in vivo. Magnetic resonance imaging (MRI) provides precise anatomic and functional assessments and is considered the gold standard in infarct assessment. ${ }^{4}$ However, the modality is time-consuming and limited in assessing physiologic aspects of the disease phenotype. ${ }^{5,6}$ Single-photon emission tomography (SPECT) with ${ }^{99 \mathrm{~m}} \mathrm{Tc}$-sestamibi imaging is also available, however, hampered by low time efficiency. ${ }^{7,8}$

Feasibility of myocardial perfusion imaging with positron emission tomography (PET) has previously been demonstrated in murine models with the ${ }^{13} \mathrm{~N}$ ammonia tracer. However, this tracer requires cyclotron and is rather time-consuming to produce, which curbs its usage. ${ }^{9-11}$ The usage of Rubidium- $82\left({ }^{82} \mathrm{Rb}\right)$ in the clinical setting is well established and could prove to be an alternative perfusion tracer in small animals despite its long positron range compared to other frequently used cardiac PET tracers. ${ }^{12}$ The high cost of the ${ }^{82} \mathrm{Rb}$-generator potentially limits its use in preclinical research. However, a generator can only be used clinically up to a maximum of 42 days after calibration. At this time point, these clinically expired generators still elute enough ${ }^{82} \mathrm{Rb}$ to perform preclinical MPI-PET for another 5 to 6 weeks. This setup can keep the price of the tracer for preclinical research at a reasonable level. The short half-life of ${ }^{82} \mathrm{Rb}$ permits fast sequential perfusion imaging and high imaging throughput ${ }^{12}$ compared to other imaging techniques such as MRI.

Therefore, the aim of our study was to explore the feasibility of ${ }^{82} \mathrm{Rb}$-imaging in rats undergoing left anterior descending artery (LAD) ligation in a dedicated small-animal PET scanner. We hypothesized that rat myocardial perfusion uptake of ${ }^{82} \mathrm{Rb}$ correlate with MRI-derived left ventricular ejection fraction (LVEF) and infarct size and predicts segmental wall motion and late gadolinium enhancement (LGE).

\section{METHODS}

All animal experiments were approved by the Danish Animal Experiments Inspectorate (Permit No. 2012-15-293400064). The animals were cared for at core animal facilities at $21 \pm 2{ }^{\circ} \mathrm{C}$ with a $12: 12$ hours dark:light cycle, were weighed once daily, and had access to water and rodent food ad libitum. The animals were acclimatized for 5 to 12 days before being included. For study design see Figure 1.

\section{Study Outline}

A total of 29 male Sprague-Dawley rats, 6 to 7 weeks old, weighing 256 grams [interquartile range (IQR): 243; 263] were divided into two groups: 18 with permanent proximal LAD ligation and 11 with ischemia duration for 35 minutes followed by reperfusion. The study design is shown in Figure 1. Myocardial infarction (MI) was induced as described elsewhere. $^{13}$ In brief, anesthesia was commenced with $4 \%$ sevoflurane and continued at $2.5 \%$ to $3 \%$. Rats were intubated and ventilated (UNO micro-ventilator-03, Netherlands) and were placed on a heated surface with ECG monitoring. The rats were pain treated with Buprenorphine $0.05 \mathrm{mg} / \mathrm{kg}$ at the intervention and three times daily the next 3 days. Thoracotomy was performed under surgical standards at the fourth intercostal space. The pericardium was opened, and the LAD ligated caudal of its origin with a 6-0 polypropylene suture. The ischemia-reperfusion rats underwent a transient LAD ligation, where the suture was looped and tighten around a polyethylene tubing (diameter $2.7 \mathrm{~mm}$ ). The ligature was released and the tubing removed after the ischemia period (35 minutes). Ischemia was confirmed by discoloration of the myocardium and ECG changes. Reperfusion was visually confirmed. The chest, muscle layers, and skin were closed with vicryl 4.0 suture. Sham operation was performed on two rats as described above except no LAD ligation took place. 


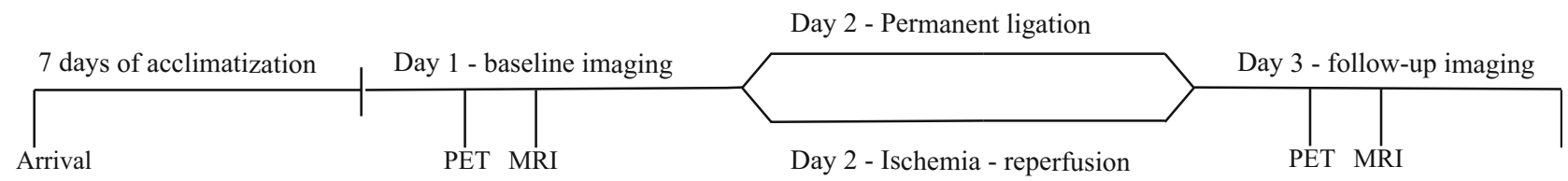

Figure 1. Study design. Arrival, of animals to the core laboratory; PET, positron emission tomography; MRI, magnetic resonance imaging.

\section{PET Imaging}

All animals underwent a baseline scan one-day prior and a follow-up scan one-day after the intervention, respectively. Imaging was performed on a dedicated preclinical PET/computed tomography (CT) scanner (Siemens Inveon, Knoxville, TN, USA). Rats were anesthetized with sevoflurane $4 \%$ in a chamber and maintained with inhaled $2 \%$ to $3.5 \%$ sevoflurane mixed with air/oxygen; the dorsal tail vein was cannulated with a permanent catheter. The rats were placed in prone position on water heated imaging bed with ECG, respiration and temperature monitoring. An initial scout CT imaging insured correct positioning. A newly expired clinical ${ }^{82} \mathrm{Rb}$ generator (CardioGen-82, Bracco Diagnostics Inc., Monroe Township, NJ, USA) was calibrated according to vendor requirements and equipped with a 3-way valve to control amount of infusion to the rats. Approximately 100 mega Becquerel (MBq) of ${ }^{82} \mathrm{Rb}$ in 1.25 to $1.5 \mathrm{~mL}$ saline solution was administered with simultaneous list mode PET acquisition for 5 minutes. For attenuation correction and anatomical coregistration, a CT scan was acquired subsequently. In addition, two rats underwent CT with contrast (Omipaque $350 \mathrm{mg} / \mathrm{mL}$, $1.5 \mathrm{~mL}$ intravenously) to validate the use of the non-contrastenhanced $\mathrm{CT}$ in the remaining rats for correct anatomical alignment of the ${ }^{82} \mathrm{Rb}$-PET examination. PET list-mode data were histogrammed into two static timeframes, the first 45 seconds and the remaining 255 seconds. The latter timeframe was used for the analyses presented in this paper. This was done to eliminate ${ }^{82} \mathrm{Rb}$-activity from the blood pool and minimize the spillover effect. The images were reconstructed non-gated using the vendor supplied ordered Poison-maximum a posteriori probability (OP-MAP)/3D-ordered subset expectation maximization (OSEM) algorithm with $3 \mathrm{~mm}$ requested resolution, 2 iterations, and 18 subsets, respectively. Images were scatter, attenuation and prompt-gamma corrected. $^{13}$

\section{PET Image Analysis}

PET data were processed and analyzed in PMOD cardiac tool version 3.3 (PMOD technologies LLC, Zurich, Switzerland). The images were first re-orientated into short, vertical, and horizontal long-axis. Next, the myocardial contour was determined in a semi-automatic manner by applying marker guidance on basal and apical points of the left ventricle (LV). The markers were placed according to extrapolation, and by fusion of PET with CT examination with and without i.v. contrast where the basal and apical points were visualized. The contours produced by the software were then assessed visually by an experienced operator (AG) and corrected manually if needed. ${ }^{82} \mathrm{Rb}$-uptakes were indexed to maximum uptake. Subsequently, polar maps of the LV were created and segmented into basal, mid and apical sections similar to the American Heart Association (AHA) 17-segment model. ${ }^{14}$ To examine the intra-observer variability, were all rat data reexamined on two separate days. All data analysis was performed blinded to treatment.

\section{MRI Imaging}

MRI was performed within an hour from PET imaging using a 7 Tesla preclinical scanner (Bruker Pharmascan, Bruker Medical, Ettlingen, Germany) with a horizontal bore and a $60-\mathrm{mm}$ transmitter-receiver coil. The rats were transferred from the PET scanner (or vice versa) in anesthesia onto a bed with similar design and monitoring as described with the PET imaging. Scout imaging ( 3 slices, $1 \mathrm{~mm}$ thickness, field of view $3.5 \times 3.5 \mathrm{~mm}^{2}$, repetition time (TR) $85 \mathrm{~ms}$, echo time (TE) $1.5 \mathrm{~ms}$ ) was utilized to verify correct positioning, and to produce 2- and 4-chamber long axis images. Short axis images were acquired perpendicular to the 2- and 4-chamber images. Next, a stack of retrospectively self-gated (Intragate) FLASH (fast low-angle shot) cine-sequence in short axis view (perpendicular to the 2- and 4-chamber images) was used to cover the entire heart in 1-mm-thick slices with 0.5 -mm gap in between. Field of view was $4.5 \times .4 .5 \mathrm{~cm}^{2}$ with a $256 \times 256$ matrix size, TR $4.8 \mathrm{~ms}$, TE $2.0 \mathrm{~ms}$, bandwidth $4566.7 \mathrm{~Hz}, 150$ number of repetitions, flip-angle $10^{\circ}$. We obtained 15 phases during the cardiac cycle. Fifteen minutes after administration of gadolinium contrast (diethylenetriamine pentacetic acid, $0.1 \mathrm{~mol} / \mathrm{kg}$, Gadovist, Bayer Scherring Pharma), a multislice ECG-gated FLASH sequence was acquired to delineate the enhanced areas in the LV at end-diastolic phase of the ECG with respiratory gating. The sequence parameters were as follows: slice thickness $1 \mathrm{~mm}$, slice gap $0.5 \mathrm{~mm}$, field of view $6 \times 6 \mathrm{~cm}^{2}$, matrix size $256 \times 256$, TR $70 \mathrm{~ms}$, TE $2.8 \mathrm{~ms}$, flipangle $30^{\circ} .15$

\section{MRI Image Analysis}

The short-axis cine images were used to calculate LVEF, end-diastolic (EDV), and end-systolic volumes (ESV), as well as wall-motion and wall-thickening on AHA-17 segments. To obtain this, the largest (EDV) and smallest LV (ESV) volume were determined manually on the 15 framed cardiac cycle; the endo- and epicardial contours of these frames were manually traced using dedicated software (CVI42 v. 4.0.1, Circle Cardiovascular Imaging, Calgary, Canada). Polar maps were generated automatically from the software. 
The endo- and epicardial contours of the LGE images were manually traced. Infarct size was assessed on follow-up LGE images and defined as hyperintensive myocardium 5 standard deviations above the mean value in a region-of-interest in normal myocardium. Isolated hypointensive areas within the enhanced region were considered as part of the infarct size. Infarct size was expressed as percent of the LV volume. The operator (AG) was blinded to PET data. To examine the inter-observer variability, a second operator $(\mathrm{KK})$ analyzed 10 randomly selected rat datasets (blinded to the results of AG).

\section{Statistics}

Continuous variables are presented as median [interquartile range, IQR] and categorical data as frequencies or percent (\%). For comparison, the non-parametric Wilcoxon matchedpairs signed rank was used. Spearman's correlation was used when comparing global outcomes between the two modalities. Linear mixed models were used for the comparison on segmental level in order to account for correlation between segments belonging to the same animal. $R^{2}$ was calculated as described by Nakagawa et al ${ }^{16}$ A two-sided $P$ value $<0.05$ was considered significant. All statistical analyses were performed using SPSS $^{\circledR}$ version 19 (IBM SPSS, Chicago, IL, USA).

\section{RESULTS}

The typical PET/CT acquisition time was 10 to 15 minutes. Baseline and follow-up PET and MRI imaging were performed on average 23 hours before and after the intervention, respectively. See Table 1.

\section{Image Quality}

Representative images of pre- and post-intervention of ${ }^{82} \mathrm{Rb}$-uptake are shown in Figure 2. The images were of average to good diagnostic quality, i.e., ability to identify the LV walls and MI. However, a pattern of better image quality was observed after the intervention, probably due to dilatation of the LV. Average total true counts per image were $60 \times 10^{6}\left[50 \times 10^{6} ; 71 \times 10^{6}\right]$ (Table 2).

\section{PET and MRI Results}

Rats in the permanent ligation group had a modest, but significant decrease in total LV myocardial ${ }^{82} \mathrm{Rb}$ uptake at follow-up compared to baseline. The apical segment showed a significant reduction in ${ }^{82} \mathrm{Rb}$-uptake for both the permanent ligation $(80.1 \%$ [73.4; 90.3] vs. $89.9 \%$ [87.2; 91.7], $P<0.005)$ and the ischemia-reperfusion $(84.9 \%$ [79.5; 88.5] vs. $88.2 \%$ [87.3; 89.4], $P=0.02)$ groups at follow-up compared to baseline imaging. See Table 3.
Table 1. Baseline characteristics

\section{RAT}

\begin{tabular}{|c|c|}
\hline \multirow[t]{3}{*}{$\mathrm{N}$} & 29 \\
\hline & PL 18 \\
\hline & IR 11 \\
\hline Age, weeks [IQR] & $6.8[6.4 ; 7.1]$ \\
\hline Weight, grams & $256[243 ; 263]$ \\
\hline Tibia length, mm & $46.9[45.4 ; 47.5]$ \\
\hline $\begin{array}{l}\text { Baseline, time from PET to MRI, } \\
\text { hh:mm }\end{array}$ & $0: 57$ [0:40; $1: 40]$ \\
\hline Baseline PET to ligation, hh:mm & $23: 30[22: 23 ; 25: 05]$ \\
\hline $\begin{array}{l}\text { Follow-up, ligation to PET, } \\
\text { hh:mm }\end{array}$ & $23: 50$ [22:30; 25:05] \\
\hline Follow-up, PET to MRI, hh:mm & $0: 50[0: 30 ; 1: 15]$ \\
\hline $\begin{array}{l}\text { Ischemia duration hh:mm* } \\
\text { 82 Rb injected, } \mathrm{MBq}\end{array}$ & $0: 35$ [0:35; 0:35] \\
\hline Baseline & $100[72 ; 126]$ \\
\hline Follow-up & $103[88 ; 122]$ \\
\hline
\end{tabular}

Values are median [IQR] interquartile range

$P E T$, positron emission tomography; MRI, magnetic resonance imaging; $P L$, permanent ligation; $I R$, ischemiareperfusion; ${ }^{82} R b$, Rubidium-82; $M B q$, mega Becquerel

* Only for ischemia-reperfusion intervention rats

There was no difference in LVEF or LV dimensions between the permanent ligation and the ischemia-reperfusion group prior to the intervention. After the intervention, the LVEF of the permanent ligation group was reduced to $30 \%$ [23; 39], while the ischemiareperfusion group was reduced to $44 \%[37 ; 58]$, compared to $61 \%[59 ; 64]$ at baseline, $P<0.001$ and $P=0.002$, respectively. Infarct size was estimated to $34.7 \%$ [19.7; 44.5] of the LV in the permanent ligation group, while it amounted to $21.7 \%[12.0 ; 29.6]$ of the LV in the ischemia-reperfusion group. See Table 2.

The sham rats did not exhibit any changes at postintervention PET and MRI imaging.

\section{PET and MRI Comparison}

The total LV and apical segment follow-up ${ }^{82} \mathrm{Rb}$ myocardial uptake in the permanent ligation group correlated strongly with follow-up MRI-derived LVEF $(r=0.89, P<0.001$ for both parameters) and with infarct size $(r=-0.81, P<0.001$ and $r=-0.78$, $P<0.001$, respectively). See Figures 3, 4 and Table 4 . Meanwhile, in the ischemia-reperfusion group, the follow-up total $\operatorname{LV}(r=-0.61, P=0.04)$ and apical $(r=-0.66, \quad P=0.02) \quad{ }^{82} \mathrm{Rb}$-uptake $\quad$ correlated 


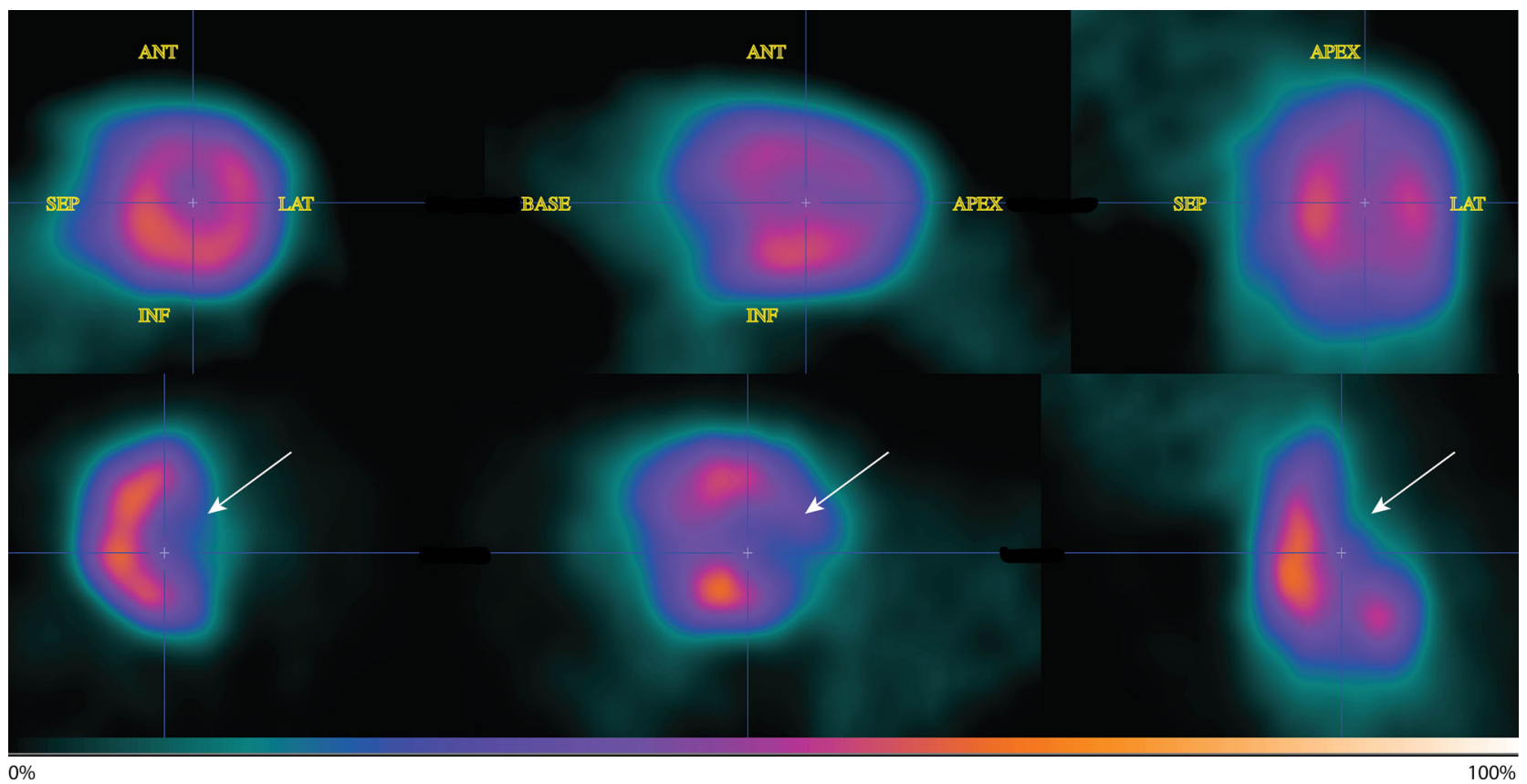

Figure 2. Upper panel baseline ${ }^{82} \mathrm{Rb}$-imaging, lower panel follow-up imaging after intervention. From left to right: short axis, horizontal and vertical view of the left ventricle of rat with permanent left anterior descending artery ligation. White arrows point to the decreased ${ }^{82} \mathrm{Rb}$-uptake at the anterolateral wall. ANT, anterior; LAT, lateral; SEP, septum; INF, inferior. Color scale indicates tracer uptake (i.e., $0 \%$ to $100 \%$ ).

Table 2. MRI parameters before and after intervention

\begin{tabular}{llll}
\hline & & MRI & \\
\cline { 2 - 4 } & Baseline imaging & Follow-up imaging & P value \\
\hline LVEF, \% [IQR] & & & \\
$\quad$ PL & $61.0[58.9 ; 63.6]$ & $30.0[23.0 ; 39.1]$ & $<0.001$ \\
IR & $60.5[58.5 ; 64.2]$ & $43.5[36.8 ; 57.8]$ & $=0.002$ \\
EDV, mL. & & & $=0.02$ \\
PL & $0.449[0.398 ; 0.505]$ & $0.519[0.470 ; 0.587]$ & ns \\
IR & $0.460[0.419 ; 0.496]$ & $0.442[0.379 ; 0.526]$ & $<0.001$ \\
ESV, mL. & & $0.395[0.314 ; 0.426]$ & $=0.004$ \\
PL & $0.175[0.142 ; 0.198]$ & $0.249[0.202 ; 0.307]$ & \\
IR & $0.177[0.156 ; 0.198]$ & $34.7[19.7 ; 44.5]$ & \\
Infarct size, \% of LV & & $21.7[12.0 ; 29.6]$ & \\
PL & na. & & \\
IR & & &
\end{tabular}

Values are median [IQR] interquartile range

$M R I$, magnetic resonance imaging; $L V E F$, left ventricle ejection fraction; $E D V$, end-diastolic volume; $E S V$, end-systolic volume; $P L$, permanent ligation; $I R$, ischemia-reperfusion; $n a$, not available; $n s$, non-significant

moderately with follow-up infarct size. In contrast, the total $\mathrm{LV}{ }^{82} \mathrm{Rb}$-uptake did not correlate significantly with follow-up LVEF.

\section{Segmental PET and MRI Comparison}

Comparing follow-up ${ }^{82} \mathrm{Rb}$-uptake according to the AHA-17 segments with follow-up MRI derivatives of 
Table 3. Indexed ${ }^{82} \mathrm{Rb}$ uptake in rat apical, mid, and basal segments of the heart before and after intervention

\begin{tabular}{lccl}
\hline $\mathbf{8 2}$ Rb-uptake & Baseline imaging & Follow-up imaging & P value \\
\hline Total & & & \\
PL & $93.6[92.0 ; 94.9]$ & $87.6[83.1 ; 94.3]$ & $=0.01$ \\
IR & $92.8[92.3 ; 93.6]$ & $91.7[89.3 ; 94.0]$ & $\mathrm{ns}$ \\
Apical & & & \\
PL & $89.9[87.2 ; 91.7]$ & $80.1[73.4 ; 90.3]$ & $=0.005$ \\
IR & $88.2[87.3 ; 89.4]$ & $84.9[79.5 ; 88.5]$ & $=0.02$ \\
Mid & $93.6[92.7 ; 95.5]$ & $87.9[82.1 ; 94.3]$ & $=0.007$ \\
PL & $93.1[91.5 ; 93.9]$ & $91.2[89.6 ; 93.8]$ & $=\mathrm{ns}$ \\
IR & $96.2[95.6 ; 97.6]$ & $93.5[91.5 ; 96.6]$ & $=0.02$ \\
Basal & $96.2[95.9 ; 97.0]$ & $96.8[95.6 ; 97.7]$ & $=\mathrm{ns}$ \\
PL & & & \\
IR & & & \\
\hline
\end{tabular}

Values are median [IQR] interquartile range

${ }^{82} R b$, rubidium-82; $P L$, permanent ligation; $I R$, ischemia-reperfusion; $n s$, non-significant

wall motion and LGE transmurality per AHA-17 segments yielded also strong associations: in the permanent ligation group, a higher ${ }^{82} \mathrm{Rb}$-uptake was associated with higher wall motion $(\beta=0.034$, CI $[0.028 ; 0.039]$, $\left.P<0.001, R^{2}=0.30\right)$, and inversely associated with lower degree of LGE transmurality $(\beta=-2.4[-2.6$; 2.2], $P<0.001, R^{2}=0.59$ ). The associations were similar when comparing the ischemia-reperfusion group ${ }^{82} \mathrm{Rb}$-uptake with the same MRI parameters, see Table 5 and Figure 5.

\section{Reproducibility}

The intra-observer interclass correlation coefficient was above 0.8 for all parameters. A subset of 10 randomly selected rat MRI data was read by two readers (AG \& KK). The inter-observer interclass correlation coefficients were between 0.76 and 0.98 , see Table 6 .

\section{DISCUSSION}

In this study, we investigated the feasibility of ${ }^{82} \mathrm{Rb}$ PET in small-animal heart models of permanent and transient ischemia and compared the PET results with MRI derivatives. The results suggest that despite the high positron range, ${ }^{82} \mathrm{Rb}$-perfusion imaging of the postischemic phase is feasible in rats and that it is correlated with LVEF, infarct size, wall motion and LGE transmurality derived from MRI.

Developing and testing novel cardioprotective treatment requires translation from preclinical validation. The small-animal heart model offers a relatively inexpensive and logistical manageable setup, where markers such as infarct size, myocardial perfusion defect, and LVEF can measure the efficacy of new treatment regiments. Thus, reliable, repeatable and timeefficient non-invasive measurements of the myocardium in rodents are valuable. ${ }^{11}$ Considering our results and the high degree of intra- and inter-observer reproducibility, the ${ }^{82} \mathrm{Rb}$-imaging could prove to be a valuable tool in evaluating myocardial perfusion in rats and allow assessment of large number of scans in a time-efficient manner. However, further studies are needed to establish the repeatability of ${ }^{82} \mathrm{Rb}$-perfusion imaging in small animals.

The combination of rapid heart rate, respiration, and thin myocardial wall combined with the long positron range of the ${ }^{82} \mathrm{Rb}$ makes assessment of the rat heart challenging due to intrinsic blurring and activity spillover from the blood pool. ${ }^{10}$ Furthermore, absolute blood flow quantification requires time-activity curves for the blood pool and the myocardium, which is difficult due to the contamination of activity across the compartments. The visual quality of the ${ }^{82} \mathrm{Rb}$ images was moderate, but nonetheless, allowed clear delineation of the myocardial wall and perfusion defects, which were strongly correlated with the MRI parameters (see Figure 3). However, applying cardiac and/or respiratory gating may partly compensate the lower image quality. Our experience (unpublished) with gating is that it seems to result in low signal-to-noise ratio images due to low count density. Administrating higher doses of radioactivity could potentially compensate this issue. However, two problems would arise: (1) higher doses 


\section{Basal}

Mid

Apical

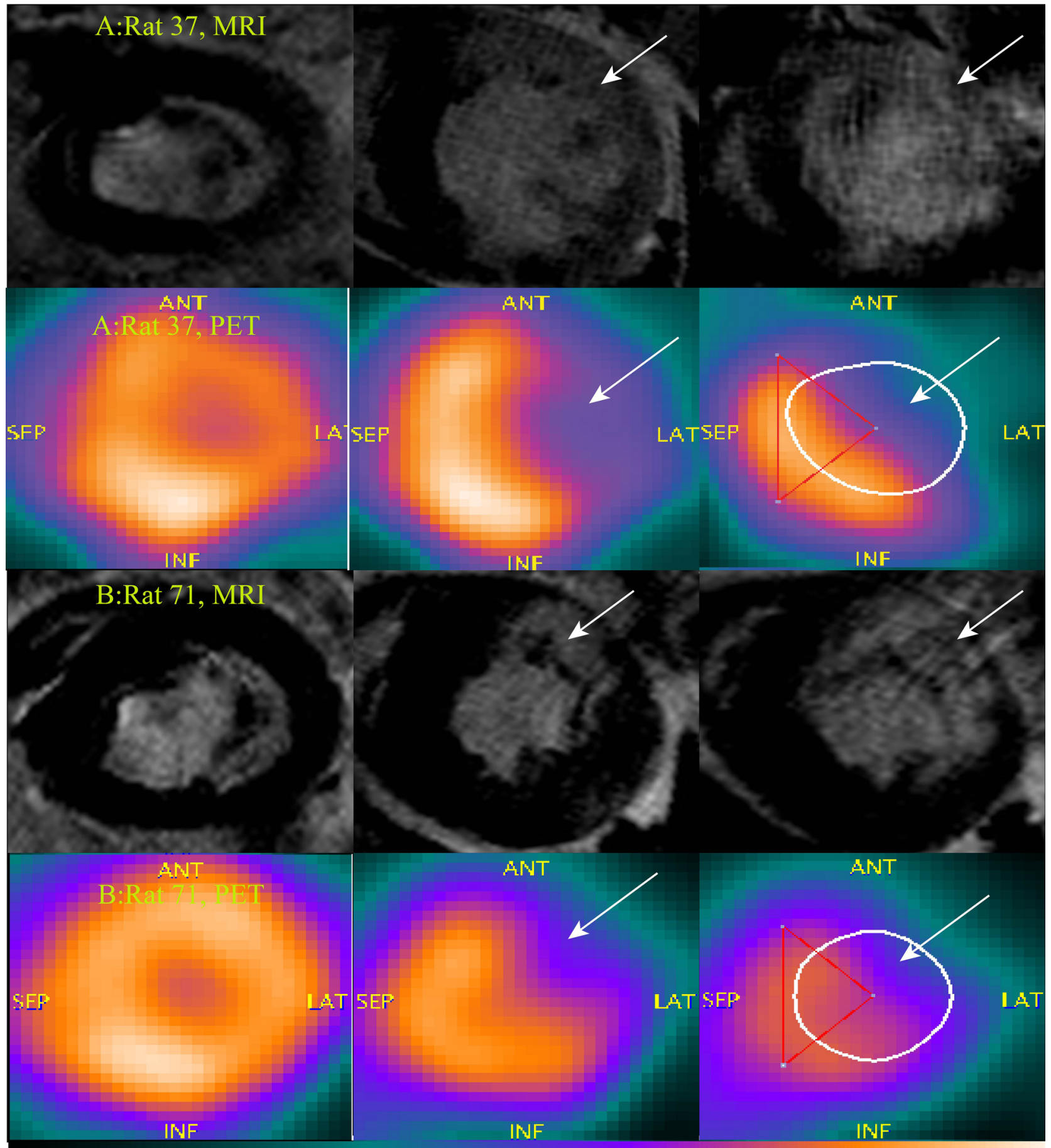

Figure 3. Comparison between MRI and ${ }^{82} \mathrm{Rb}-\mathrm{PET}$. Two upper panels $\mathbf{A}$ represent basal, mid and apical short-axis view of the left ventricle of a rat with permanent ligation with MRI and PET. Two lower panels $\mathbf{B}$ represent a rat with ischemia-reperfusion. White arrows point to areas with late gadolinium enhancement on the MRI and decreased ${ }^{82} \mathrm{Rb}$-uptake on PET images. ANT, anterior; $L A T$, lateral; SEP, septum; INF, inferior Color scale indicates tracer uptake (i.e., $0 \%$ to $100 \%$ ). 
Permanent ligation Rb-82 PET uptake and MRI infarct size

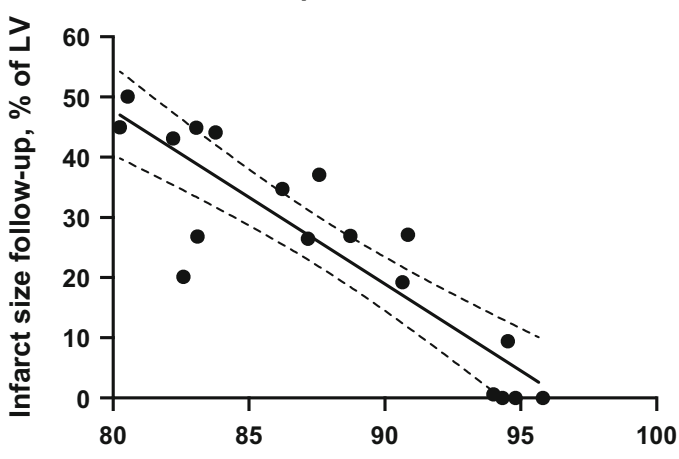

LV relative perfusion follow-up, \%

Ischemia - reperfusion

Rb-82 PET uptake and MRI infarct size

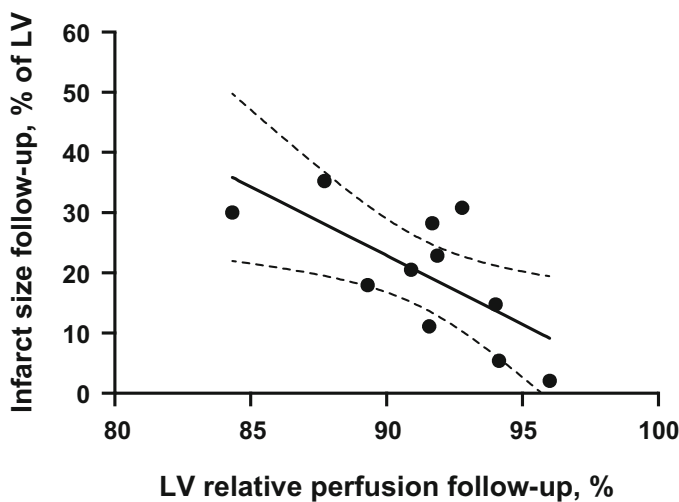

Permanent ligation Rb-82 PET uptake and MRI LVEF

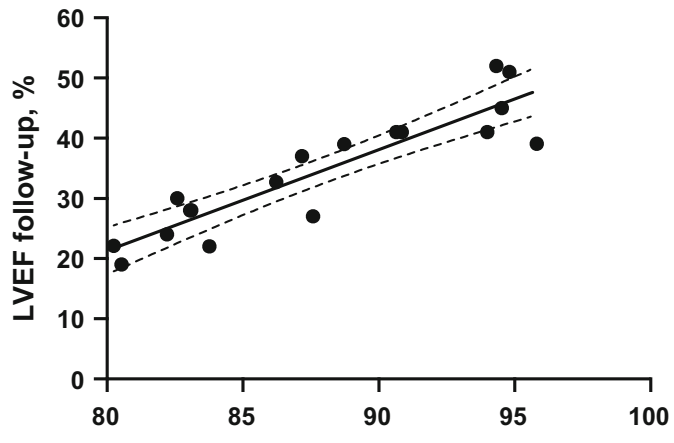

$\mathrm{LV}$ relative perfusion follow-up, $\%$ Ischemia - reperfusion Rb-82 PET uptake and MRI LVEF

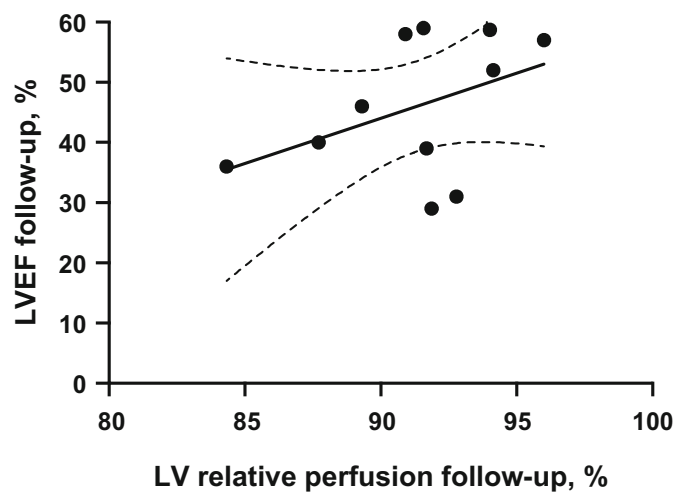

Figure 4. Upper panel permanent ligated rats. Lower panel ischemia-reperfusion rats. Global ${ }^{82} \mathrm{Rb}$-uptake and MRI-derived infarct size, and global ${ }^{82} \mathrm{Rb}$-uptake and MRI-derived left ventricle ejection fraction. ${ }^{82} R b$, rubidium-82; $M R I$, magnetic resonance imaging; $L V E F$, left ventricle ejection fraction.

Table 4. Correlation between ${ }^{82} \mathrm{Rb}$ uptake and post-intervention MRI

\begin{tabular}{lll}
\hline & \multicolumn{2}{c}{ MRI follow-up } \\
\cline { 2 - 3 } $\mathbf{8 2} \mathbf{R b}$-uptake follow-up & \multicolumn{1}{c}{ LVEF, $\%$} & \multicolumn{1}{c}{ Infarct size, $\%$} \\
\hline Total & & \\
PL & $r=0.89, P<0.001$ & $r=-0.81, P<0.001$ \\
IR & $r=0.19, P=\mathrm{ns}$ & $r=-0.61, P=0.04$ \\
Apical & $r=0.89, P<0.001$ & $r=-0.78, P<0.001$ \\
PL & $r=0.40, P=\mathrm{ns}$ & $r=-0.66, P=0.02$ \\
IR & $r=0.91, P<0.001$ & $r=-0.85, P<0.001$ \\
Mid & $r=0.28, P=\mathrm{ns}$ & $r=-0.60, P=\mathrm{ns}$ \\
PL & $r=0.44, P=\mathrm{ns}$ & $r=-0.34, P=\mathrm{ns}$ \\
IR & $r=-0.16, P=\mathrm{ns}$ & $r=-0.36, P=\mathrm{ns}$ \\
Basal &
\end{tabular}

$L V E F$, left ventricle ejection fraction; $P L$, permanent ligation; $I R$, ischemia-reperfusion; $n s$, non-significant 
Table 5. Linear Mixed Models

\begin{tabular}{|c|c|c|c|c|c|c|c|c|c|}
\hline \multirow{3}{*}{$\begin{array}{l}\text { Outcome } \\
\text { PET }\end{array}$} & \multicolumn{9}{|c|}{ Rat MRI follow-up no explanatory factors except PET derivatives } \\
\hline & \multicolumn{3}{|c|}{$\begin{array}{c}\text { Wall thickening } \\
\text { (\% AHA-17 segments) }\end{array}$} & \multicolumn{3}{|c|}{$\begin{array}{c}\text { Wall Motion } \\
\text { (mm AHA-17 segments) }\end{array}$} & \multicolumn{3}{|c|}{$\begin{array}{c}\text { LGE (\% transmurality } \\
\text { per AHA-17 segments) }\end{array}$} \\
\hline & Beta & $\boldsymbol{P}$ & $\boldsymbol{R}^{2}$ & Beta & $\boldsymbol{P}$ & $\boldsymbol{R}^{2}$ & Beta & $\boldsymbol{P}$ & $\boldsymbol{R}^{\mathbf{2}}$ \\
\hline $\begin{array}{l}\text { Total LV } \\
{ }^{82} \text { Rb-uptake } \\
\text { PL group (\%) }\end{array}$ & $\begin{array}{c}0.32 \\
{[0.02 ; 0.61]}\end{array}$ & $0.04 *$ & 0.01 & $\begin{array}{c}0.034 \\
{[0.028 ; 0.039]}\end{array}$ & $0.001^{*}$ & 0.30 & $\begin{array}{c}-2.4 \\
{[-2.6 ;-2.2]}\end{array}$ & 0.001 * & 0.59 \\
\hline $\begin{array}{l}\text { Total LV } \\
{ }^{82} \mathrm{Rb} \text {-uptake } \\
\text { IR group (\%) }\end{array}$ & $\begin{array}{c}0.4 \\
{[-0.08 ; 0.89]}\end{array}$ & ns & 0.01 & $\begin{array}{c}0.05 \\
{[0.04 ; 0.06]}\end{array}$ & $0.001^{*}$ & 0.34 & $\begin{array}{c}-2.7 \\
{[-3.2 ;-2.2]}\end{array}$ & $0.001^{*}$ & 0.35 \\
\hline
\end{tabular}

$L V E F$, left ventricle ejection fraction; $P L$, permanent ligation; $I R$, ischemia-reperfusion; $n s$, non-significant; $A H A$, American Heart Association; $L G E$, late gadolinium enhancement

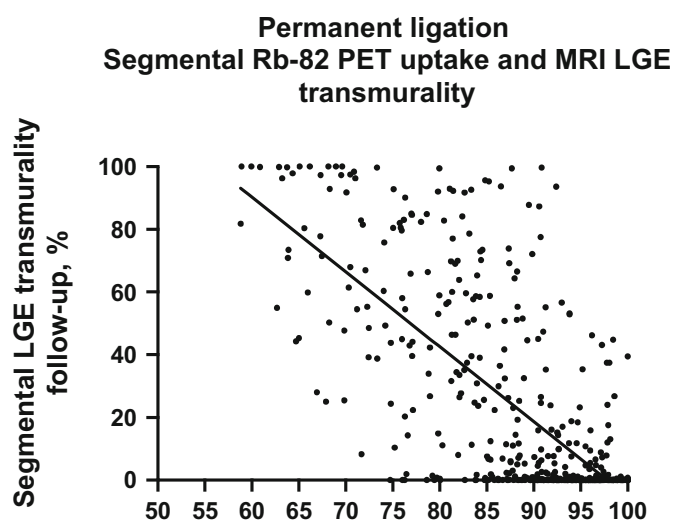

Segmental relative perfusion follow-up, \%

Ischemia-reperfusion

Segmental Rb-82 PET uptake and MRI LGE transmurality

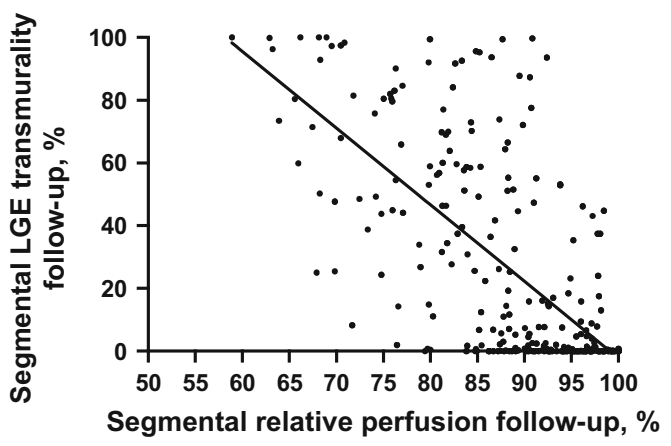

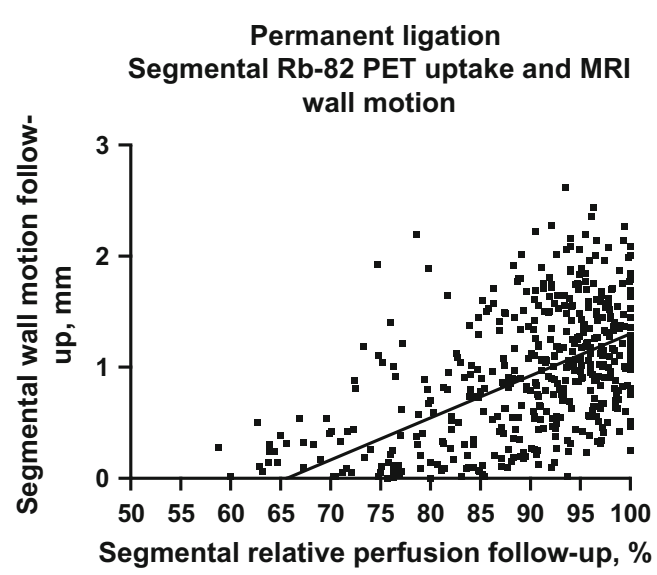

Ischemia-reperfusion Segmental Rb-82 PET uptake and MRI wall motion

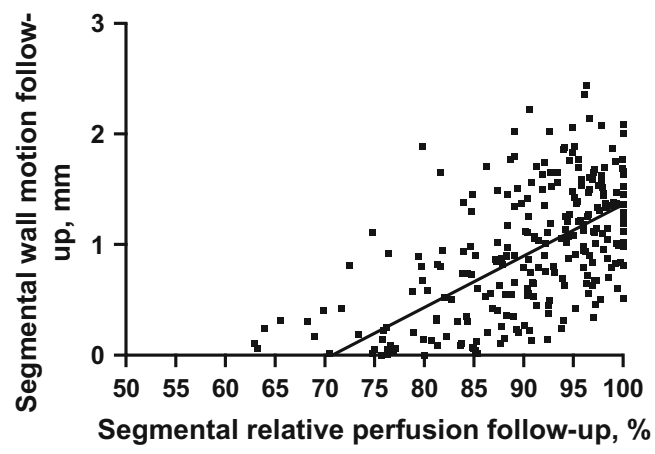

Figure 5. Upper panel permanent ligated rats. Lower panel ischemia-reperfusion rats. Segmental ${ }^{82} \mathrm{Rb}$-uptake and MRI late gadolinium enhancement, and segmental ${ }^{82} \mathrm{Rb}$-uptake and MRI wall motion. ${ }^{82} R b$, rubidium-82; $M R I$, magnetic resonance imaging; $L G E$, late gadolinium enhancement; $\mathrm{mm}$, millimeters. 
Table 6. Inter-observer and intra-observer correlation

\begin{tabular}{|c|c|c|c|}
\hline MRI data & LVEF baseline & LVEF follow-up & Infarct size \\
\hline $\begin{array}{l}\text { Inter-observer ICC } \\
\text { (10 datapoints) }\end{array}$ & $\begin{array}{l}0.810 \text { [0.237;0.953] } \\
P=0.01\end{array}$ & $\begin{array}{l}0.981 \text { [0.904;0.996] } \\
\quad P<0.001\end{array}$ & $\begin{array}{l}0.767[0.163 ; 0.953] \\
P=0.04\end{array}$ \\
\hline PET data & $\begin{array}{l}\text { Total LV }{ }^{82} \text { Rb-uptake } \\
\text { baseline }\end{array}$ & $\begin{array}{l}\text { Total LV }{ }^{82} \text { Rb-uptake } \\
\text { follow-up }\end{array}$ & $\begin{array}{c}\text { Basal }{ }^{82} \text { Rb-uptake } \\
\text { post }\end{array}$ \\
\hline $\begin{array}{l}\text { Intra-observer ICC } \\
\text { (29 datapoints) }\end{array}$ & $\begin{array}{c}0.899 \text { [0.786;0.953] } \\
P<0.001\end{array}$ & $\begin{array}{l}0.899[0.788 ; 0.952] \\
\quad P<0.001\end{array}$ & $\begin{array}{c}0.828 \text { [0.639; } 0.918] \\
P<0.001\end{array}$ \\
\hline
\end{tabular}

Inter-observer and intra-observer (ICC) = interclass correlation for MRI and PET data

require larger elution volumes precluding the use in small animals. (2) higher doses would also increase random and scatter effects, in addition to greater deadtime artifacts. The Inveon scanner user guide recommends no higher injected dose than 100 to $120 \mathrm{MBq}$ in order to obtain the best noise equivalent count rate (NECR). However, further investigation into post-imaging reconstruction and processing is warranted.

It could be speculated that the reduced ${ }^{82} \mathrm{Rb}$-uptake in the infarcted regions is the result of the residual partial volume effect caused by the thinning of the myocardial wall after infarction. However, while the anterolateral wall of many rats had diminished wall motion as observed by MRI cine, no outright wall thinning was observed at the follow-up MRI scans.

MRI was selected as reference due to its high spatial resolution and high reproducibility in LV function and morphology estimations. ${ }^{17}$ Furthermore, MRI allows assessment of the infarct size with LGE, which has been validated against histology in rats. ${ }^{15}$ MRI-derived parameters are associated with morbidity and mortality in the clinical setting, which justifies MRI as Ref. ${ }^{18}$ As expected, we found larger infarcts in the permanent ligation group compared to the ischemia-reperfusion group. Our results were very similar to the non-invasive and histological estimations of Luo et al for both intervention methods, ${ }^{15}$ which in combination with the high degree of inter-observer reproducibility, supports the validity of our MRI results. For both the permanent ligation and the ischemia-reperfusion group, the correlations for follow-up ${ }^{82} \mathrm{Rb}$-uptake were strongest with the MRI-derived infarct sizes as in contrast to the LVEF. This suggests that the ${ }^{82} \mathrm{Rb}$ activity is a better surrogate marker of infarct size than LV function. In contrast to the permanent ligation group, the follow-up ${ }^{82} \mathrm{Rb}$-uptake of the ischemia-reperfusion group did not significantly correlate to follow-up LVEF. This was probably the result of the limited reduction of ${ }^{82} \mathrm{Rb}$ activity at followup due to variation in the degree and extent of ischemia applied. Furthermore, the relative limited resolution and blurring impede the detection of small perfusion defects. Hence, underscoring the need for improved post-imaging processing to reducing blurring and bloodmyocardium cross-contamination.

${ }^{13} \mathrm{~N}$-ammonia perfusion tracer has previously been used successfully in rats and mice to depict perfusion defects. $^{9,19}$ However, both studies only conducted imaging when the artery was totally occluded and not after ischemia-reperfusion. While ${ }^{13} \mathrm{~N}$-ammonia produces images with better spatial resolution compared to ${ }^{82} \mathrm{Rb}$ because of less scatter and shorter positron range, ${ }^{82} \mathrm{Rb}$-imaging has two main advantages: first, no need of onsite cyclotron, and second, a higher throughput due to a faster imaging protocol. However, the continuing cost of an ${ }^{82} \mathrm{Rb}$-generator must be kept in mind.

${ }^{82} \mathrm{Rb}$-imaging has become the standard perfusion tracer in many PET centers and has been validated against other perfusion tracers and histology. ${ }^{20-22}$ However, important inherent traits of ${ }^{82} \mathrm{Rb}$ are unknown regarding tracer kinetics and extraction during hyperemia, and metabolic derangement after ischemiareperfusion injury. ${ }^{23}$ Furthermore, the impact of many standard medication regiments in coronary artery disease (e.g., statins, antihypertensive, anticoagulants) on ${ }^{82} \mathrm{Rb}$-uptake is unknown. Nevertheless, our model seems promising and with further improvement in reconstruction processing could enable preclinical testing with various drugs and improve the pathophysiological understanding of the ${ }^{82} \mathrm{Rb}$-uptake in myocardial tissue free of injury and after infarction.

\section{LIMITATIONS}

This study has several limitations. One being that preconditioning of the heart in the clinical setting plays an important role but was absent in our setup. Our model of ischemia-reperfusion is comparable to the clinical setting, but does not adequately address the 
preconditioning issue. However, consecutive opening and closing of the snare before total occlusion can simulate this effect. Second, the in vivo results were not compared to ex vivo measurements, which could validate the methodology further. However, the MRI, which was used as reference in this study, has previously been validated with histology. ${ }^{15}$

Despite the calibration of the ${ }^{82} \mathrm{Rb}$-generator before each use, the exact amount of tracer activity injected in each rat is difficult to estimate because of the manual handling of the dose infusion and rapid radioactivity decay. Moreover, the species-specific myocardial uptake of the ${ }^{82} \mathrm{Rb}$ in rats is unknown and could be different from humans. However, ${ }^{82} \mathrm{Rb}$ blood flow measurements investigated in canine models were comparable with microsphere blood flow measurements. ${ }^{23}$

\section{CONCLUSION}

To the best of our knowledge, this study is the first to examine the feasibility of ${ }^{82} \mathrm{Rb}$-PET imaging and comparing it to MRI-derived measurements in rats. In a chronic and an ischemia-reperfusion rat model, the infarct size correlated significantly with the ${ }^{82} \mathrm{Rb}$-uptake. Furthermore, the segmental ${ }^{82} \mathrm{Rb}$-uptake after infarction predicted the wall motion and LGE enhancement as measured by MRI. Further studies are needed to define the accuracy, precision, and repeatability of ${ }^{82} \mathrm{Rb}$-perfusion imaging in rats. These results may with enhanced post-imaging processing encourage preclinical serial evaluation of the myocardial perfusion in a timeefficient manner.

\section{NEW KNOWLEDGE GAINED}

${ }^{82} \mathrm{Rb}$-PET can be utilized to produce myocardial perfusion images in rats, and enhance the cost-benefit profile of clinically available ${ }^{82} \mathrm{Rb}$-generator.

\section{Acknowledgements}

This study received support from the Research Grant Committee of Rigshospitalet Copenhagen University Hospital, Copenhagen, Denmark, No: E-22160-07 (Dr. Adam Ali Ghotbi).

\section{Disclosure}

The authors that have no conflict of interest.

\section{Open Access}

This article is distributed under the terms of the Creative Commons Attribution 4.0 International License (http://creativecommons.org/licenses/by/4.0/), which permits unrestricted use, distribution, and reproduction in any med- ium, provided you give appropriate credit to the original author(s) and the source, provide a link to the Creative Commons license, and indicate if changes were made.

\section{References}

1. Schwartz Longacre L, Kloner RA, Arai AE, et al. New horizons in cardioprotection: Recommendations from the 2010 national heart, lung, and blood institute workshop. Circulation 2011;124:1172-9. doi:10.1161/CIRCULATIONAHA.111.032698.

2. Abarbanell AM, Herrmann JL, Weil BR, et al. Animal models of myocardial and vascular injury. J Surg Res 2010;162:239-49. doi: 10.1016/j.jss.2009.06.021.

3. Redfors B, Shao Y, Omerovic E. Myocardial infarct size and area at risk assessment in mice. Exp Clin Cardiol 2012;17:268-72.

4. Thomas D, Bal H, Arkles J. Noninvasive assessment of myocardial viability in a small animal model: Comparison of MRI, SPECT, and PET. Magn Reson Med 2008;59:252-9. doi:10.1002/ mrm.21445.Noninvasive.

5. Higuchi T, Nekolla SG, Jankaukas A, et al. Characterization of normal and infarcted rat myocardium using a combination of small-animal PET and clinical MRI. J Nucl Med 2007;48:288-94.

6. Protti A, Sirker A, Shah AM, Botnar R. Late gadolinium enhancement of acute myocardial infarction in mice at 7T: Cine-FLASH versus inversion recovery. J Magn Reson Imaging 2010;32:878-86. doi:10.1002/jmri.22325.

7. Wollenweber T, Zach C, Rischpler C, et al. Myocardial perfusion imaging is feasible for infarct size quantification in mice using a clinical single-photon emission computed tomography system equipped with pinhole collimators. Mol Imaging Biol 2010;12: 427-34. doi:10.1007/s11307-009-0281-5.

8. Liu Z, Kastis GA, Stevenson GD, et al. Quantitative analysis of acute myocardial infarct in rat hearts with ischemia-reperfusion using a high-resolution stationary SPECT system. J Nucl Med 2002;43: 933-9.

9. Kudo T, Fukuchi K, Annala AJ, et al. Noninvasive measurement of myocardial activity concentrations and perfusion defect sizes in rats with a new small-animal positron emission tomograph. Circulation 2002;106:118-23. doi:10.1161/01.CIR.0000020221. 28996.78.

10. Inubushi M, Jordan MC, Roos KP, et al. Nitrogen-13 ammonia cardiac positron emission tomography in mice: Effects of clonidineinduced changes in cardiac work on myocardial perfusion. Eur J Nucl Med Mol Imaging 2004;31:110-6. doi:10.1007/s00259-003-1328-5.

11. Stegger L, Hoffmeier A-N, Schäfers KP, et al. Accurate noninvasive measurement of infarct size in mice with high-resolution PET. J Nucl Med 2006;47:1837-44.

12. Ghotbi AA, Kjær A, Hasbak P. Review: Comparison of PET rubidium-82 with conventional SPECT myocardial perfusion imaging. Clin Physiol Funct Imaging 2013;34:163-70. doi:10.1111/ cpf. 12083

13. Clemmensen AE, Ghotbi AA, Bodholdt RP, et al. Perfusion imaging using rubidium-82 (82Rb) PET in rats with myocardial infarction: First small animal cardiac 82Rb-PET. J Nucl Cardiol 2016;. doi:10.1007/s12350-016-0564-6.

14. Cerqueira MD. Standardized Myocardial Segmentation and Nomenclature for Tomographic Imaging of the Heart: A Statement for Healthcare Professionals From the Cardiac Imaging Committee of the Council on Clinical Cardiology of the American Heart Association. Circulation 2002;105:539-42. doi:10.1161/hc0402.10 2975. 
15. Luo D, Yao YY, Li YF, et al. Myocardial infarction quantification with late gadolinium-enhanced magnetic resonance imaging in rats using a 7-T scanner. Cardiovasc Pathol 2012;21:112-9. doi: 10.1016/j.carpath.2011.03.005.

16. Nakagawa $S$, Schielzeth $H$. A general and simple method for obtaining R2 from generalized linear mixed-effects models. Methods Ecol Evol 2013;4:133-42. doi:10.1111/j.2041-210x. 2012.00261.x

17. Stuckey D, Carr C, Tyler D, Clarke K (2008) Cine-MRI vs. 2Dechocardiography to measure left ventricular function in rat heart in vivo. In: Proc 16th Sci Meet Int Soc Magn Reson Med Toronto: 1029.

18. El Aidi H, Adams A, Moons KGM, et al. Cardiac magnetic resonance imaging findings and the risk of cardiovascular events in patients with recent myocardial infarction or suspected or known coronary artery disease: A systematic review of prognostic studies. J Am Coll Cardiol 2014;63:1031-45. doi:10.1016/j.jacc.2013. 11.048.

19. Inubushi M, Wu JC, Gambhir SS, et al. Positron-emission tomography reporter gene expression imaging in rat myocardium.
Circulation 2003;107:326-32. doi:10.1161/01.CIR.0000044 385.60972.AE.

20. Germino M, Ropchan J, Mulnix T, et al. Quantification of myocardial blood flow with $82 \mathrm{Rb}$ : Validation with $15 \mathrm{O}$-water using time-of-flight and point-spread-function modeling. EJNMMI Res 2016;6:68. doi:10.1186/s13550-016-0215-6.

21. deKemp RA, Klein R, Beanlands RSB. 82Rb PET imaging of myocardial blood flow-have we achieved the 4 " $R$ "'s to support routine use? EJNMMI Res 2016;6:69. doi:10.1186/s13550016-0225-4.

22. Chatal J-F, Rouzet F, Haddad F, et al. Story of Rubidium-82 and advantages for myocardial perfusion PET imaging. Front Med 2015;2:65. doi:10.3389/fmed.2015.00065.

23. Lekx KS, deKemp RA, Beanlands RS, et al. Quantification of regional myocardial blood flow in a canine model of stunned and infarcted myocardium: comparison of rubidium- 82 positron emission tomography with microspheres. Nucl Med Commun 2010;31: 67-74. doi:10.1097/MNM.0b013e328332b32a. 